Удк 343.9:519.87

DOI 10.17150/2500-4255.2018.12(2).230-236

\title{
ЦИФРОВАЯ КРИМИНОЛОГИЯ: МАТЕМАТИЧЕСКИЕ МЕТОАЫ ПРОГНОЗИРОВАНИЯ (ЧАСТЬ 1)
}

\author{
А.П. Суходолов ${ }^{1}$, С.В. Иванцов ${ }^{2,}$, Т.В. Молчанова ${ }^{2}$, Б.А. Спасенников ${ }^{3}$, М.А. Калужина ${ }^{3,4}$ \\ ${ }^{1}$ Байкальский государственный университет, г. Иркутск, Российская Федерация \\ ${ }^{2}$ Московский университет МВД России им. В.Я. Кикотя, г. Москва, Российская Федерация \\ ${ }^{3}$ Научно-исследовательский институт Федеральной службы исполнения наказаний России, г. Москва, \\ Российская Федерация \\ ${ }^{4}$ Кубанский государственный университет, г. Краснодар, Российская Федерация
}

\author{
Информация о статье \\ Дата поступления \\ 28 ноября 2017 г. \\ Дата принятия в печать \\ 6 апреля 2018 г. \\ Дата онлайн-размещения \\ 27 апреля 2018 г.

\section{Ключевые слова} \\ Цифровая криминология; \\ математическое прогнозирование; \\ математические методы; \\ преступность; методы \\ прогнозирования преступности; \\ математическая модель \\ преступности; параметры оценки \\ преступности; профилактика \\ преступлений; Байкальский \\ государственный университет
}

\begin{abstract}
Аннотация. Статья посвящена рассмотрению проблем цифровой криминологии, анализу методов математического прогнозирования и возможности их использования в области изучения преступности. Усложнение задачи противодействия преступности обусловливает необходимость как критического осмысления существующих методов, так и изыскания возможностей выхода за рамки традиционных методов изучения правовых явлений. Информационно-аналитическая деятельность органов внутренних дел, основанная на разработке программ предупреждения преступности, имеет своей главной целью применение математических методов анализа преступности. Предметом изучения выступает совокупность математических методов, отобранных с учетом целесообразности их применения для криминологического прогнозирования. Авторы выделяют следующие методы: метод моделирования, корреляционный анализ, анализ ранговых корреляций и таблиц сопряженности, дискриминантный анализ, регрессионный анализ, дисперсионный анализ, ковариационный анализ, факторный анализ, анализ временных рядов, метод сезонных колебаний, метод максимального правдоподобия (в частности, его разновидность - метод наименьших квадратов), метод расчета среднегодовых темпов прироста, аппарат логических решающих функций, распознавание образов, вариационные исчисления, спектральный анализ, цепи Маркова, алгебра логики и др. Математическое прогнозирование в цифровой криминологии состоит в использовании имеющихся количественных и качественных параметров преступности, получении их математической зависимости от времени, пространства, других известных независимых переменных. В результате исследования установлено, что использование математической обработки криминологической информации позволяет увеличить точность прогнозных оценок. Статья подготовлена в рамках развития научной школы цифровой экономики Байкальского государственного университета.
\end{abstract}

\section{DIGITAL CRIMINOLOGY: MATHEMATICAL METHODS OF PREDICTION (PART 1)}

\author{
Alexander P. Sukhodolov', Sergey V. Ivantsov', 3 , Tatiana V. Molchanova ${ }^{2}$, \\ Boris A. Spasennikov ${ }^{3}$, Marina A. Kaluzhina ${ }^{3,4}$ \\ ${ }^{1}$ Baikal State University, Irkutsk, the Russian Federation \\ ${ }^{2}$ Moscow University of the Ministry of the Interior of Russia named after V.Y. Kikot, Moscow, the Russian Federation \\ ${ }^{3}$ Research Institute of the Federal Penitentiary Service of Russia, Moscow, the Russian Federation \\ ${ }^{4}$ Kuban State University, Krasnodar, the Russian Federation
}

\section{Article info}

Received

2017 November 28

Accepted

2018 April 6

Available online

2018 April 27

\section{Keywords}

Digital criminology; mathematical

forecasting; mathematical methods;

\begin{abstract}
The paper discusses the problems of digital criminology, analyses the mathematical prediction methods and the possibilities of using them in the sphere of crime research. The growing complexity of the task of crime counteraction makes it necessary both to critically reconsider the existing methods and to go beyond the constraints of the traditional methods of researching legal phenomena. The information and analytical work of the internal affairs' bodies, based on developing the programs of crime prevention, has the primary goal of using mathematical methods of crime analysis. The object of the research is a set of mathematical methods selected on the basis of their suitability for making criminological predictions. The authors single out the following methods: the modeling method, the correlation analysis, the analysis of rank correlations and conjugacy tables, discriminant analysis, regression analysis, variance analysis, covariance analysis, factor analysis, time series analysis, seasonal oscillation method, maximum like-
\end{abstract}


crime; crime prediction methods; mathematical model of criminality; parameters of crime estimation; crime prevention; Baikal State University lihood method (in particular, its variety - least squares method), average annual growth rate calculation method, logical decision functions, pattern recognition, Markov chains, algebra of logic, etc. The mathematical prediction in digital criminology consists in using the existing qualitative and quantitative parameters of crime, in calculating their mathematical dependence on time, space and other known independent variables. The study allowed the authors to determine that the processing of criminological information using the mathematical methods increases the accuracy of predictions. The paper is prepared within the framework of developing the research school of digital economy of Baikal State University.
Каждая наука лишь тогда достигает совершенства, когда породнится с математикой. И. Кант

В эпоху цифровой экономики усиливается потребность определить будущее, предвидеть, предсказать, предвосхитить, предугадать, спрогнозировать результаты социальных, политических и экономических перемен. Различия в горизонте прогностической деятельности в значительной мере обусловливают выбор метода прогнозирования. Познание тенденций и закономерностей развития криминологических явлений основывается на применении научных методов исследования, в том числе математических, которые занимают особое место в прогнозировании, цифровой криминологии.

Вопрос использования математических методов в общественных науках является на сегодня достаточно актуальным. В 50-х гг. прошлого столетия выдающийся кибернетик Н. Винер отметил: «Кибернетика - ничто, если математика не служит ей опорой, если не inesse, то in posse. И математическая социология, и математическая экономика, или эконометрика, страдают от неправильного понимания того, как следует применять математический аппарат в общественных науках и чего вообще можно ожидать от применения математических методов» [1, с. 96].

У истоков математического прогнозирования в отечественной криминологии стояли выдающиеся криминологи Г.А. Аванесов, С.Е. Вицин, Л.В. Кондартюк, В.С. Овчинский, К.К. Горяинов, М.П. Клейменов и др. Вместе с тем современный период развития научной мысли диктует необходимость поиска новых возможностей постижения криминологических и социальноправовых явлений, а именно приложения математических закономерностей к изучаемым явлениям или процессам. Математика обладает универсальным характером, и потому ее достижения применимы ко многим отраслям знаний.

Теоретическое понимание криминологической действительности в значительной сте- пени зависит от выбранных методов анализа. Криминологическая наука, исследуя преступность, ее отдельные виды и группы, личность преступника, составляя портрет жертвы преступления и определяя группы риска, изучает факторы, детерминирующие преступность, в их массовом статистическом наблюдении. Анализ, обобщение и интерпретация этих факторов немыслимы без использования статистических и математических методов исследования. Установление взаимосвязи и взаимозависимости внутри преступности, формирование ожидаемого социального образа наиболее опасного преступника с выделением различных его типологических характеристик и степени их влияния на отдельные криминогенные процессы, определение наиболее вероятного сценария развития криминогенной ситуации в контексте ожидаемого влияния комплекса детерминирующих факторов носят не динамический, а статистический (корреляционный) характер. Нахождение и установление подобного рода взаимосвязей невозможны без применения математических методов. Криминологическим проблемам цифровой экономики было посвящено несколько публикаций во «Всероссийском криминологическом журнале» за 20172018 гг. Они послужили началом разработки теории цифровой криминологии.

Прогнозирование - наиболее сложный вид деятельности в системе криминологических исследований. Прогнозирование преступности, без которого невозможна заблаговременная подготовка к эффективной предупредительной деятельности, может осуществляться на основе статистического и математического изучения тенденций и закономерностей преступности. Подобного рода исследование возможно лишь на основе объективного знания фактического состояния преступности и реальных результатов работы системы правоохранительных органов. Для этого на постоянной основе должно решаться две группы задач. 
Первая группа: реальная оценка криминологической обстановки (текущей, годовой, многолетней), определение основных тенденций преступности и ее различных видов, установление взаимосвязей между криминологическими показателями и сведениями о социальных, экономических, демографических, политических, правовых процессах в регионе и в целом в стране.

Вторая группа: реальная оценка деятельности правоохранительных органов, определение и корректировка главных направлений правоохранительной деятельности, а также деятельности иных субъектов власти по минимизации причин и условий, оказывающих существенное влияние на преступность.

Решению данных задач способствует комплексный статистический анализ преступности, который может использоваться как способ прогнозирования будущей криминогенной обстановки и как инструмент обоснования краткосрочных и долгосрочных управленческих решений, поэтому математическое изучение преступности представляет собой один из способов опережающего анализа развития криминогенной обстановки.

Интенсификация информационно-аналитической деятельности органов внутренних дел, обусловленная разработкой и внедрением программ предупреждения преступности, имеет своей главной целью применение математических методов анализа преступности в их деятельности.

Математическое описание криминологической обстановки в конкретном регионе предусматривает ее рассмотрение в контексте познания прошлого, настоящего и будущего состояния преступности. Следует отметить, что математические методы прогнозирования имеют высокую достоверность получаемых результатов.

Разработка прогноза и связанные с ним построение и экспериментальная верификация вероятностно-статистической модели, как правило, основаны на одновременном использовании:

- информации о природе и сущности анализируемого явления, представленной обычно в виде тех или иных теоретических закономерностей, гипотез;

- статистических данных, характеризующих процесс и результаты функционирования анализируемого явления или системы.

В настоящее время наукой выработаны различные методы и модели прогнозирования. Метод прогнозирования представляет собой алгоритм действий, которые необходимо совершить для получения модели прогнозирования. Понятие «метод прогнозирования» гораздо шире понятия «модель прогнозирования». Модель прогнозирования - это функциональное представление, соразмерно описывающее изучаемый процесс и являющееся основой для получения его будущих значений. В прогнозировании преступности наиболее целесообразно применение моделей временных рядов.

Модели временных рядов - математические модели, которые стремятся найти зависимость будущего значения от прошлого внутри самого процесса и на установленной зависимости вычислить прогноз. Данные модели универсальны для различных предметных областей, т.е. их общий вид не меняется в зависимости от природы временного ряда. Модели временных рядов можно разделить на две группы: статистические (регрессионные модели - линейная регрессия, нелинейная регрессия; авторегрессионные модели; модель экспоненциального сглаживания; модель по выборке максимального подобия и т.д.) и структурные (нейросетевые модели; модели на базе цепей Маркова; модели на базе классификационно-регрессионных деревьев) [2, р. 54-72].

Математических моделей прогнозирования достаточно много, но чаще всего в тех или иных вариациях они имеют свойство повторения.

Используя вышеуказанные модели, специалисты в области математики классифицируют все методы прогнозирования на две группы - интуитивные и формализованные [3, с. 12].

Интуитивные методы прогнозирования имеют дело с суждениями и иными различными оценками экспертов. Сегодня данные методы [4] часто применяются в экономике, политике, так как система, состояние которой необходимо спрогнозировать, или очень сложна и не поддается математическому описанию, или очень примитивна и не нуждается в таком описании. К такого рода методам в криминологии относятся индивидуальные и коллективные оценки, метод интервью, метод анкетирования, метод комиссий, аналитические докладные записки и т.д. [5, с. 45].

Формализованные методы - это методы прогнозирования, в процессе использования которых строят модели прогнозирования, т.е. определяют такую математическую зависимость, которая позволяет вычислить будущее значение процесса, иными словами, сделать прогноз. Таким образом, формализованные методы основа- 
ны на математическом прогнозировании и представляют собой математические методы.

Математические методы в криминологическом прогнозировании, как правило, являются неотьемлемой частью более глубокого статистического, социологического, сравнительного и исторического анализа криминологических данных в целях их системного и модульного анализа, моделирования криминологических процессов, выявления упорядоченности и типологии объектов изучения, оценки надежности статистической информации и др. [6, с. 23].

Математические методы в зависимости от вида математического описания объектов прогнозирования и способов определения неизвестных параметров условно подразделяются на методы моделирования процессов, описываемых дифференциальными уравнениями, и методы экстраполяции, или статистические (Statistical method of forecasting).

Математическое прогнозирование в криминологии заключается в использовании имеющихся параметров прогнозируемого объекта (количественная характеристика преступности, которая является постоянной в течение периода основания и периода упреждения прогноза или принимается за таковую), обработке статических данных о преступности, личности преступника математическими методами, получении их математической зависимости от времени, пространства и других известных независимых переменных и вычислении с помощью найденной зависимости характеристик объекта в заданный момент времени при заданных значениях других независимых переменных [7, с. 256].

Сегодня появились и закрепились на практике такие интеллектуальные методы и специализированные инструменты, как, например, DataMining. Понятие DataMining переводится как добыча данных, извлечение информации, раскопка данных, интеллектуальный анализ данных, средства поиска закономерностей, извлечение знаний, анализ шаблонов, «извлечение зерен знаний из гор данных», раскопка знаний в базах данных, информационная проходка данных, «промывание» данных. Это технология, которая предназначена для поиска в больших объемах данных неочевидных (сомнительных), объективных и важных (полезных) в практической деятельности закономерностей. Неочевидных предполагается, что найденные закономерности не обнаруживаются стандартными (классическими) методами обработки информации или мне- нием экспертов. Объективных - проявившиеся закономерности должны полностью соответствовать действительности, в отличие от экспертного мнения, которое в значительной степени субъективное. Практически полезных - это значит, что выводы имеют конкретное значение, которому можно найти практическое применение [8, p. 210-212; 9, р. 114-116].

Главное достоинство названной технологии заключается в том, что большинство статистических методов для выявления взаимосвязей в анализируемых данных используют концепцию усреднения по выборке, приводящую к операциям над несуществующими величинами, тогда как DataMining оперирует реальными значениями.

DataMining - мультидисциплинарная область, формирующаяся на базе таких наук, как прикладная статистика, распознавание образов, искусственный интеллект, теория баз данных и др. В криминологическом исследовании это процесс обнаружения в сырых данных о преступности ранее неизвестных, практически полезных и доступных для интерпретации знаний, необходимых для принятия управленческих решений в деятельности по предупреждению преступлений.

Методы математического прогнозирования отличаются объективностью, достоверностью и точностью получаемых результатов, но при условии правильного выбора математической модели [10, р. 140-162].

Математическое прогнозирование состоит из следующих этапов:

- сбор и подготовка исходных данных;

- выбор и обоснование математической модели прогнозируемого объекта (статистико-математический инструментарий);

- обработка статистических данных о преступности для установления неизвестных параметров модели;

- выполнение расчетов, количественный и качественный анализ полученных результатов.

К математическим методам относятся методы моделирования, корреляционный анализ, анализ ранговых корреляций и таблиц сопряженности, дискриминантный анализ, регрессионный анализ, дисперсионный анализ, ковариационный анализ, факторный анализ, анализ временных рядов, метод сезонных колебаний, метод максимального правдоподобия (в частности, его разновидность - метод наименьших квадратов), метод расчета среднегодовых темпов прироста, аппарат логических решающих функций, распознавание образов, вариацион- 
ные исчисления, спектральный анализ, цепи Маркова, алгебра логики, теория игр и др. [11; $12 ; 13$, с. 26-30]. Все они объединены тем, что представляют собой различные подходы к решению главной проблемы многомерного статистического анализа - проблемы статистического исследования зависимостей, которая является основой криминологического прогнозирования.

Исследователи в области криминологического прогнозирования чаще всего используют методы математической экстраполяции, математического моделирования, метод исторических аналогий [14, р. 240-256; 15, р. 457-459].

Среди них наибольшее распространение получил метод математической экстраполяции, который позволяет количественно оценить прогнозируемые процессы в криминологии. Он основан на изучении сложившихся в прошлом тенденций и закономерностей преступности и распространения их в будущем. Так, согласно данному методу, в общественной, социально-экономической жизни действует принцип инерции, т.е. наблюдаемые тенденции и закономерности достаточно устойчивы и могут проявляться в течение некоторого периода.

Прогнозная экстраполяция (Prognostische Extrapolation) осуществляется с помощью выравнивания статистических рядов вне их связи с другими рядами динамики, влияние которых учитывается в усредненном виде лишь на основе предшествующих данных о состоянии преступности. Предпосылка о сохранении неизменности условий предшествующего периода при экстраполяции ограничивает возможности применения этого метода сравнительно непродолжительными периодами, в течение которых не происходит существенных качественных колебаний. Наиболее объективны выводы прогнозирования при соотношении продолжительности предшествующего периода (ретроспекции) и периода упреждения (проспекции - времени от настоящего в будущее, на которое разрабатывается прогноз). Некоторые исследователи используют понятие «прогнозный горизонт» [7, с. 256], характеризующийся максимально возможным периодом упреждения прогноза. Для применения данного метода необходимо иметь продолжительный ряд показателей преступности за предшествующий период. Система метода экстраполяции состоит из метода подбора функции, метода усреднения, метода адаптивного сглаживания. Процесс прогнозирования осуществляет- ся с помощью описания трендовой модели, или, иначе, методом экстраполяции тренда.

Трендоваямодель - это математическая модель, описывающая изменение прогнозируемого или анализируемого показателя только в зависимости от времени: $y=f(t)$. Можно утверждать, что это один из инертных методов прогнозирования, и называется он «наивным» прогнозом, так как предполагает инерционность развития, которая представляется в виде проектирования прошлых тенденций в будущее, а главное независимость показателей развития от тех или иных факторов [15, р. 457-459].

Главной аналитической задачей, которая решается при исследовании рядов динамики с помощью экстраполяции, выступает выявление скрытых тенденций и закономерностей развития преступности. Для установления общей тенденции формирования преступности берутся сглаженные расчетные значения. Преобразованный с помощью метода скользящей средней динамический ряд (временная последовательность ретроспективных и перспективных значений переменной объекта прогнозирования) отражает общую тенденцию развития преступности. Некорректность данного метода заключается в том, что не всегда возможно (а если и возможно, то с особой осторожностью) переносить различные проявления тенденций преступности, которые сформировались в прошлом, на будущее, поскольку в перспективе может измениться сила воздействия многих факторов на формирование преступности.

В прогнозной экстраполяции используются следующие ее виды: прогнозирование по функции с гибкой структурой (Forecastingusing the function offlexible structure), метод экспоненциального сглаживания (Method of exponentials moothing), метод гармонических весов (Method of harmonics cales).

Рассматривая природу использования анализа временных рядов в прогнозировании преступности, отметим, что выбранному типу анализа соответствует кратко- и среднесрочный прогноз. Построение же долгосрочного прогноза предполагает необходимость применения метода статистического анализа специальных знаний экспертов. Наряду с экстраполяцией в криминологическом изучении преступности используют метод прогнозной интерполяции (Prognostics Interpolation). Данный метод основан на математической интерполяции, при которой выбор интерполирующей функции осу- 
ществляется с учетом условий и ограничений развития объекта прогнозирования (в нашем случае - преступности).

Изучение динамических рядов преступности позволит решить следующие аналитические задачи: установление динамики, характера, интенсивности изменения преступности за исследуемый период; определение средней интенсивности изменения преступности за весь рассматриваемый период; установление скрытых закономерностей развития преступ- ности. Выявление общих и частных тенденций в уровне и структуре преступности будет способствовать становлению и развитию цифровой криминологии.

Итак, использование математической обработки криминологической информации позволяет увеличить точность прогнозных оценок, что будет рассмотрено во второй части настоящей статьи, посвященной проблемам криминологического прогнозирования [16], цифровой криминологии.

\section{СПИСОК ИСПОЛЬЗОВАННОЙ ЛИТЕРАТУРЫ}

1. Винер Н. Творец и робот: обсуждение некоторых проблем, в которых кибернетика сталкивается с религией / Н. Винер. - М. : Прогресс, 1966. - 104 c.

2. Jingfei Y. Power System Short-term Load Forecasting: Thesis for Ph.d degree / Y. Jingfei. — Darmstadt : Elektrotechnik und Informationstechnik der Technischen Universitat, 2006. - 139 p.

3. Чучуева И.А. Модель прогнозирования временных рядов по выборке максимального подобия : дис... канд. техн. наук : 05.13 .18 / И.А. Чучуева. - М., 2012. - 154 с.

4. Armstrong J.S. Forecasting for Marketing / J.S. Armstrong // Quantitative Methods in Marketing. - London : International Thomson Business Press, 1999. - P. 92-119.

5. Математические методы в социальных науках / ред. П. Лазарсфельд, Н. Генри. - М. : Прогресс, 1973. - 354 с.

6. Блувштейн Ю.Д. Криминология и математика / Ю.Д. Блувштейн. - М. : Юрид. лит., 1974. - 176 с.

7. Бестужев-Лада И.В. Социальное прогнозирование. Курс лекций / И.В. Бестужев-Лада, Г.А. Наместникова. - М. : Пед. о-во России, 2001. - 392 с.

8. Sutherland E.H. Principles of Criminology / E.H. Sutherland, D.R. Cressey. - Chicago : Lippincott, 1960. - 646 p.

9. Fox V. Introduction to Criminology / V. Fox. - New Jersey : Prentice Hall, 1976. - $462 \mathrm{p}$.

10. Taylor I. The New Criminology: For a Social Theory of Deviance / I. Taylor, P. Walton, J. Young. - London : Routledge, 1973. $-325 \mathrm{p}$.

11. Brantingham P.J. A Conceptual Model of Crime Prevention / P.J. Brantingham, F.L. Faust // Crime and Delinquency. 1976. - Vol. 22, iss. 3. - P. 284-296.

12. Спасенников Б.А. Актуальные проблемы уголовного права: обзор литературы / Б.А. Спасенников // Актуальные вопросы образования и науки. - 2015. - № 1-2 (47-48). - С. 36-38.

13. Спасенников Б.А. Актуальные проблемы уголовного права : учеб. пособие / Б.А. Спасенников. - Архангельск : Ин-т упр., 2013. -255 c.

14. Williams K.S. Textbook on Criminology / K.S. Williams. - Oxford : Oxford Univ. Press, 2012. - 680 p.

15. The Encyclopedia of Police Science / ed. Jack R. Greene. $-3^{\text {rd }}$ ed. - New York : Routledge, 2007. - Vol. 1 : A-I index. -717 p.

16. Суходолов А.П. Цифровая экономика и цифровая криминология / А.П. Суходолов, Б.А. Спасенников // Прорывные научные исследования: проблемы, закономерности, перспективы : сб. ст. 10-й Междунар. науч.-практ. конф. - Пенза : Наука и просвещение, 2018. - С. 224-226.

\section{REFERENCES}

1. Viner N. Tvorets i robot: obsuzhdenie nekotorykh problem, v kotorykh kibernetika stalkivaetsya s religiei [The Creator and the Robot: Discussing the Issues Where Cybernetics Meets Religion]. Moscow, Progress Publ., 1966. 104 p.

2. Jingfei Y. Power System Short-term Load Forecasting: Thesis for Ph.D degree. Darmstadt, Elektrotechnik und Informationstechnik der Technischen Universitat, 2006. 139 p.

3. Chuchueva I.A. Model' prognozirovaniya vremennykh ryadov po vyborke maksimal'nogo podobiya. Kand. Diss. [The model of predicting time series for choosing the maximum semblance. Kand. Diss.]. Moscow, 2012. 154 p.

4. Armstrong J.S. Forecasting for Marketing. Quantitative Methods in Marketing. London, International Thomson Business Press, 1999, pp. 92-119.

5. Lazarsfeld P., Henry N. (eds.). Readings in Mathematical Social Sciences. Chicago, Science Research Associates, 1966. 371 p. (Russ. ed.: Lazarsfeld P., Henry N. (eds.). Matematicheskie metody v sotsial'nykh naukakh. Moscow, Progress Publ., 1973. 354 p.).

6. Bluvshtein Yu.D. Kriminologiya i matematika [Criminology and Mathematics]. Moscow, Yuridicheskaya literatura Publ., 1974. $176 \mathrm{p}$

7. Bestuzhev-Lada I.V., Namestnikova G.A. Sotsial'noe prognozirovanie [Social Prediction]. Moscow, Pedagogical Society of Russia Publ., 2001. 392 p.

8. Sutherland E.H., Cressey D.R. Principles of Criminology. Chicago, Lippincott, 1960. 646 p.

9. Fox V. Introduction to Criminology. New Jersey, Prentice Hall, 1976. 462 p.

10. Taylor I., Walton P., Young J. The New Criminology: For a Social Theory of Deviance. London, Routledge, 1973.325 p.

11. Brantingham P.J., Faust F.L. A Conceptual Model of Crime Prevention. Crime and Delinquency, 1976, vol. 22, iss. 3, pp. 284-296.

Russian Journal of Criminology, 2018, vol. 12, no. 2, pp. 230-236 
12. Spasennikov B.A. Topical issues of criminal law: an overview of publications. Aktual'nye voprosy obrazovaniya i nauki $=$ Topical Issues of Education and Science, 2015, no. 1-2 (47-48), pp. 36-38. (In Russian).

13. Spasennikov B.A. Actual problems of criminal law. Arkhangelsk, Institute of Management Publ., 2015. 300 p.

14. Williams K.S. Textbook on Criminology. Oxford, Oxford University Press, 2012. 680 p.

15. Greene J.R. (ed.). The Encyclopedia of Police Science. $3^{\text {rd }}$ ed. New York, Routledge, 2007. Vol. 1. 717 p.

16. Sukhodolov A.P., Spasennikov B.A. Digital economy and digital criminology. Proryvnye nauchnye issledovaniya: problemy, zakonomernosti, perspektivy. Sbornik statei 10-i Mezhdunarodnoi nauchno-prakticheskoi konferentsii [Breakthrough Research: Issues, Regularities, Prospects. Collected articles for the $10^{\text {th }}$ Research Conference]. Penza, Nauka i prosveshchenie Publ., 2018, pp. 224-226. (In Russian).

\section{ИНФОРМАЦИЯ ОБ АВТОРАХ}

Суходолов Александр Петрович - ректор Байкальского государственного университета, доктор экономических наук, профессор, заслуженный экономист Российской Федерации, г. Иркутск, Российская Федерация; e-mail: rector@bgu.ru.

Иванцов Сергей Вячеславович - профессор кафедры криминологии Московского университета МВД России им. В.Я. Кикотя, ведущий научный сотрудник Научно-исследовательского института Федеральной службы исполнения наказаний России, доктор юридических наук, профессор, г. Москва, Российская Федерация; e-mail: isv1970@mail.ru.

Молчанова Татьяна Витальевна - доцент кафедры криминологии Московского университета МВД России им. В.Я. Кикотя, кандидат юридических наук, доцент, г. Москва, Российская Федерация; e-mail: molcha@yandex.ru.

Спасенников Борис Аристархович - главный научный сотрудник Научно-исследовательского института Федеральной службы исполнения наказаний России, доктор юридических наук, доктор медицинских наук, профессор, г. Москва, Российская Федерация; e-mail: borisspasennikov@yandex.ru.

Калужина Марина Анатольевна - доцент кафедры криминалистики и правовой информатики Кубанского государственного университета, г. Краснодар, Российская Федерация; ведущий научный сотрудник Научно-исследовательского института Федеральной службы исполнения наказаний России, кандидат юридических наук, доцент, г. Москва, Российская Федерация; e-mail: kaluzhina. marishka@yandex.ru.

\section{ДЛЯ ЦИТИРОВАНИЯ}

Суходолов А.П. Цифровая криминология: математические методы прогнозирования (часть 1) / А.П. Суходолов, С.В. Иванцов, Т.В. Молчанова, Б.А. Спасенников, М.А. Калужина // Всероссийский криминологический журнал. 2018. - T. 12, № 2. - C. 230-236. - DOI: 10.17150/25004255.2018.12(2).230-236.

\section{INFORMATION ABOUT THE AUTHORS}

Sukhodolov, AlexanderP. - Rector, Baikal State University, Doctor of Economics, Professor, Honored Economist of the Russian Federation, Irkutsk, the Russian Federation; e-mail: rector@bgu.ru.

Ivantsov, Sergey V. - Professor, Chair of Criminology, Moscow University of the Ministry of the Interior of Russia named after V.Y. Kikot, Leading Researcher, Research Institute of the Federal Penitentiary Service of Russia, Doctor of Law, Professor, Moscow, the Russian Federation; e-mail: isv1970@mail.ru.

Molchanova, Tatiana V. - Ass. Professor, Chair of Criminology, Moscow University of the Ministry of the Interior of Russia named after V.Y. Kikot, Ph.D. in Law, Ass. Professor, Moscow, the Russian Federation; e-mail: molcha@yandex.ru.

Spasennikov, Boris A. - Chief Researcher, Research Institute of the Federal Penitentiary Service of Russia, Doctor of Law, Doctor of Medicine, Professor, Moscow, the Russian Federation; e-mail: borisspasennikov@yandex.ru.

Kaluzhina, Marina A. - Ass. Professor, Chair of Criminalistics and Law Informatics, Kuban State University, Krasnodar, the Russian Federation; Leading Researcher, Research Institute of the Federal Penitentiary Service of Russia, Ph.D. in Law, Ass. Professor, Moscow, the Russian Federation; e-mail: kaluzhina.marishka@yandex.ru.

\section{FOR CITATION}

Sukhodolov A.P., Ivantsov S.V., Molchanova T.V., Spasennikov B.A., Kaluzhina M.A. Digital criminology: mathematical methods of prediction (part 1). Vserossiiskii kriminologicheskii zhurnal = Russian Journal of Criminology, 2018 , vol. 12 , no. 2 , pp. 230-236. DOI: 10.17150/25004255.2018.12(2).230-236. (In Russian). 